\title{
FUNCTIONS OF POTENTIAL TYPE
}

\author{
BY \\ MAYNARD G. ARSOVE(1)
}

1. Introduction. It is well known $\left({ }^{2}\right)$ that the bounded signed measures on a Boolean algebra of sets form a Banach lattice, the norm being taken as the total variation. This order of ideas is clearly applicable to potential theory, since the signed Borel measure, or mass distribution, corresponding to a potential $w$ has finite total variation $\Phi(w)$. However, one is at once confronted with the unpleasant fact that a sequence $\left\{w_{k}\right\}$ of potentials may diverge uniformly to $+\infty$ while $\left\{\Phi\left(w_{k}\right)\right\}$ converges to 0 : e.g., in the plane, $w_{k}(z)$ $=\max [k,(1 / k) \log |z|]$. Thus, normed by the total variation alone, the space $P$ of potentials admits sequences whose terms ultimately disappear out of sight, but which none the less converge strongly to zero.

To avoid this situation, we employ a definition of norm making use of the values of the potentials as well as their mass distributions. In what follows we deal only with potentials on the finite plane $\mathcal{E}$, although generalizations to higher-dimensional spaces are inevitable. The norm is defined in terms of a certain class of linear functionals $F$ as

$$
\|w\|=|F(w)|+\Phi(w) .
$$

A suitable choice of $F(w)$ would be the areal mean of $w$ over a fixed disc in $\varepsilon$, but it is essential for the full generality of results to allow a much wider selection (according to Definition 5 of $\S 7$ ). Under this norm the space $P$ is incomplete, since for $c$ any positive constant the sequence of potentials

$$
w_{k}(z)=\max [c,(1 / k) \log |z|]
$$

converges strongly to the (nonpotential) function identically $c$. Moreover, even with the adjunction of all constant functions $P$ remains incomplete, so that its metric completion $P^{f}$ is nontrivial.

Another concept which we examine is that of the order of an entire $\delta$-subharmonic function, i.e. a function representable on the entire plane $\varepsilon$ as the difference of two subharmonic functions $\left({ }^{3}\right)$. This is developed along classical lines in terms of a characteristic function $T_{r}(w, z)$ introduced previously [(I),

Presented to the Society, June 21, 1952; received by the editors October 15, 1952.

(1) Based on a portion of the author's thesis, submitted in partial fulfillment of the requirements for the degree of Doctor of Philosophy at Brown University. The author wishes to express his appreciation to Professor M. H. Heins, under whose direction the thesis was written, for numerous suggestions and criticisms, and to Professor M. Brelot of the University of Grenoble for a critical reading of the manuscript.

(2) Birkhoff [1, p. 252].

${ }^{(3)}$ An introduction to the theory of $\delta$-subharmonic functions is given in Arsove [1], which we refer to in the sequel as (I). 
Definition 8]; for brevity we denote the characteristic function for $z=0$ by $T_{r}(w)$, rather than $T_{r}(w, 0)$. It is easily shown that all potentials are of order zero, and one is then led by way of generalization to the study of complete $[(\mathrm{I})$, Definition 4$]$ entire $\delta$-subharmonic functions of order zero whose mass distributions have finite total variation. These functions, which we refer to as functions of potential type, can be characterized as the complete entire $\delta$-subharmonic functions $w$ for which $\lim _{r \rightarrow \infty}\left[T_{r}(w) / \log r\right]$ exists finitely. Although they share many of the properties of potentials, functions of potential type are more numerous, and we show in fact that the class $P$ of all functions of potential type coincides with $P^{\sharp}$. The upper and lower envelopes of two functions of potential types are themselves of potential type, and we thus have the rather striking result that metric completion of $P$ results also in lattice completion.

In spite of being nonseparable, the Banach space $P$ has important properties, appearing in terms of a type of convergence weaker than the usual weak convergence. This mode of convergence, which we designate as convergence $(A)$, is defined by convergence of linear functionals of a certain class along with weak $\left(^{4}\right)$ convergence of the mass distributions $\left(^{5}\right)$. With the aid of a lemma asserting that every set of positive interior capacity supports a distribution of the unit mass having a continuous potential, we are able to establish a number of convergence theorems for $\delta$-subharmonic functions and to show that the exceptional sets which arise are of zero interior capacity. However, Choquet has proved, by means of a postulational approach to the theory of capacity, that all Borel sets are capacitable. Using this result (the details of which have not yet appeared in print), we find in fact that the exceptional sets are of zero exterior capacity. In this form our convergence theorems contain as special cases those due to Brelot and Cartan for monotone increasing sequences of subharmonic functions $\left({ }^{6}\right)$.

While a detailed study of the Banach space and $(L)$ space properties of $\mathbb{P}$ promises to be fruitful, we defer a study of this sort to a later paper and concern ourselves here primarily with the analytical aspects of the theory. We include also an outline of the theory of entire $\delta$-subharmonic functions, slightly more comprehensive than is actually needed for the theory of functions of potential type. This has been done, not only because of the intrinsic interest in extending the classical theory of entire functions to the $\delta$-subharmonic case, but also because a number of authors $\left({ }^{7}\right)$ have had use for isolated topics in this theory. Moreover, work has been done by Brelot along these lines, but independent of the classical development and without explicit use of the notions of order, exponent of convergence, and genus.

(4) See Definition 7 and subsequent remarks in (I).

(5) An exact description of convergence (A) is to be found in Definition 7 of $\S 8$.

( $)$ See Cartan $[1 ; 2]$.

(7) E.g. Transue [1], Heins [1], Inoue [1]. 
In the final section $(\$ 9)$ we indicate a correlation between functions of potential type and functional analysis. Essentially, the space $P$ can be identified abstractly as the conjugate of the Cartesian product space $\Gamma_{\infty}=R \times C_{\infty}$, where $R$ is the real line and $C_{\infty}$ is the space of all continuous functions on $\varepsilon$ vanishing at infinity. Convergence (A) then appears as weak convergence of linear functionals on $\Gamma_{\infty}$.

2. Entire $\delta$-subharmonic functions. Order. Exponent of convergence. The theory of entire $\delta$-subharmonic functions is a direct generalization, via the characteristic function of (I), of the classical theory of entire analytic and meromorphic functions. Accordingly, our discussion parallels closely the standard treatments $\left({ }^{8}\right)$. Proofs can for the most part be reconstructed from the classical arguments (with integrals replacing infinite products) and are therefore largely omitted $\left({ }^{9}\right)$.

We establish first a few preliminary results. For $w$ an entire $\delta$-subharmonic function we denote the total mass by $M(w)$ and the total variation of the mass distribution by $\Phi(w)$. Attaching a subscript $r$ to these functions will indicate, respectively, the total mass and the total variation of the mass distribution, both taken over the disc $S_{r}(0)$ of radius $r$ and center $0\left({ }^{10}\right)$. Further, we use $\mu_{r}(w)$ for the integral mean of $w$ over the circumference $C_{r}(0)$ of $S_{r}(0)$.

The quantity $\Phi_{r}(u)$ for an entire subharmonic function $u$ can be estimated, by using a formula of F. Riesz (Radó [1, 5.18 and 5.14]), as

$$
\frac{\mu_{r_{2}}(u)-\mu_{r_{1}}(u)}{\log r_{2}-\log r_{1}} \leqq \Phi_{r}(u) \leqq \frac{\mu_{r_{1}}(u)-\mu_{r_{8}}(u)}{\log r_{4}-\log r_{3}}
$$

where $r_{1}<r_{2}<r<r_{3}<r_{4}$. There results the elementary, but fundamental,

THEOREM 1. If $u$ is an entire subharmonic function, then (in the extended sense)

$$
\lim _{r \rightarrow \infty}\left[\mu_{r}(u) / \log r\right]=\Phi(u)
$$

COROLlaRY 1.1. If $u$ and $v$ are entire subharmonic functions such that $u$ dominates $v$ a.e. in the sense of angular measure on $C_{r}(0)$ for all sufficiently large $r$, then $\Phi(u) \geqq \Phi(v)$.

The interrelationship between characteristic functions and canonical envelopes [(I), Definition 8] then leads to

COROLlaRY 1.2. If $w$ is an entire $\delta$-subharmonic function, $\lambda$ any canonical

( $\left.{ }^{8}\right)$ E.g. Titchmarsh [1], Valiron [1], Nevanlinna [1].

( $\left.{ }^{9}\right)$ Brief proofs of some of the theorems are given in Arsove [2].

${ }^{\left({ }^{10}\right)}$ For the sake of simplicity we shall carry over the same notation (omitting the symbol $w)$ whenever we have occasion to deal with a mass distribution independently of a $\delta$-subharmonic function. 
envelope for $w$, and $T_{r}(w)$ any characteristic function, then

$$
(1 / 2) \Phi(w) \leqq \lim _{r \rightarrow \infty}\left[T_{r}(w) / \log r\right]=\Phi(\lambda) .
$$

Adapting from Nevanlinna [1], we define the "order" of an entire $\delta$-subharmonic function as follows.

Definition 1. By the order of an entire $\delta$-subharmonic function $w$ we mean the quantity $\rho(w)$ defined in the extended sense as

$$
\rho(w)=\lim \sup _{r \rightarrow \infty}\left[\log T_{r}(w) / \log r\right] .
$$

Since the characteristic function is uniquely determined by $w$ to within an additive constant, it is immaterial which characteristic function we use. Moreover, setting aside the trivial case of $w$ identically constant, we have $\lim _{r \rightarrow \infty} T_{r}(w)=+\infty$, so that in all cases $0 \leqq \rho(w) \leqq+\infty$.

As noted by Nevanlinna [1, pp. 207-208], there are two fundamental properties of $\rho(w)$ each of which serves to characterize it completely.

THEOREM 2. An entire $\delta$-subharmonic function w has order $\rho$ if and only if either of the following conditions holds:

(1) for any $\alpha>\rho$ and all sufficiently large $r, T_{r}(w)<r^{\alpha}$, while for any $\beta<\rho$ there exist arbitrarily large $r$ such that $T_{r}(w)>r^{\beta}$;

(2) the integral $\int_{1}^{\infty}\left[T_{r}(w) / r^{\kappa+1}\right] d r$ converges for $\kappa>\rho$ and diverges for $\kappa<\rho$.

COROLlARY 2.1. If $w$ is an entire $\delta$-subharmonic of finite order and $\kappa>\rho(w)$, then (relative to infinity) $\Phi_{r}(w)=O\left(r^{x}\right)$.

Proof. Sweeping out the mass on $N_{1}(0)$, the neighborhood of radius 1 about 0 , we find from Corollary 42.1 of (I) that

$$
\int_{0}^{r}\left[\Phi_{t}(w) / t\right] d t=2 T_{r}(w)-\mu_{r}(|w|)+\text { const. }<2 r^{\alpha}
$$

holds for large $r$. The rest of the argument is classical.

This result is closely related in the subharmonic case to (C) of Lemma 4 in Brelot's paper on the growth of subharmonic functions (Brelot [1]), although the characteristic function is there replaced by the circumferential mean.

Additional elementary properties of the order function follow from properties of the characteristic function noted in Theorem 35 of (I).

ThEOREM 3. If $w, w_{1}$, and $w_{2}$ are entire $\delta$-subharmonic functions of finite order and $c$ any nonzero real number, then (1) $\rho(c w)=\rho(w)$ and (2) $\rho\left(w_{1}+w_{2}\right)$ $\leqq \max \left[\rho\left(w_{1}\right), \rho\left(w_{2}\right)\right]$, equality holding whenever $\rho\left(w_{1}\right) \neq \rho\left(w_{2}\right)$.

The equality case of (2) is established by assuming $\rho\left(w_{1}\right)<\rho\left(w_{2}\right)$ and applying the inequality $\rho\left(w_{2}\right) \leqq \max \left[\rho\left(w_{1}+w_{2}\right), \rho\left(w_{1}\right)\right]$.

We show next that in problems dealing with entire subharmonic functions it is permissible to interchange the characteristic function and the maximum 
function in the definition of order and in the computation of the total mass of canonical envelopes.

THEOREM 4. If $u$ is an entire subharmonic function and $\sigma_{r}(u)$ the maximum of $u$ on the circular circumference $C_{r}(0)$, then

$$
\begin{aligned}
\limsup _{r \rightarrow \infty}\left[\log \sigma_{r}(u) / \log r\right] & =\limsup _{r \rightarrow \infty}\left[\log T_{r}(u) / \log r\right], \\
\lim _{r \rightarrow \infty}\left[\sigma_{r}(u) / \log r\right] & =\lim _{r \rightarrow \infty}\left[T_{r}(u) / \log r\right] .
\end{aligned}
$$

Proof. It is obvious that $T_{r}(u)=\mu_{r}\left(u^{+}\right) \leqq \sigma_{r}(u)$, while a familiar inequality on the Poisson kernel yields for $c>1$ the estimate in the reversed direction

$$
\sigma_{r}(u) \leqq[(c+1) /(c-1)] \mu_{c r}\left(u^{+}\right) .
$$

Since the maximum function for a polynomial behaves at infinity like that for the term of highest degree, we have

CoROllary 4.1. If $u$ is a subharmonic polynomial of degree $n$, then $\rho(u)=n$. order.

It then follows from Theorem 31 of (I) that every polynomial has finite

Corresponding to the concept of order of an entire $\delta$-subharmonic function there is the notion of "exponent of convergence " of its mass distribution.

Definition 2. By the exponent of convergence of (the mass distribution of) an entire $\delta$-subharmonic function $w$ we mean the quantity $\rho_{1}(w)$ defined in the extended sense as

$$
\rho_{1}(w)=\limsup _{r \rightarrow \infty}\left[\log \Phi_{r}(w) / \log r\right] .
$$

The choice of terminology here is made clear by (3) of

THEOREM 5. An entire $\delta$-subharmonic function w has exponent of convergence $\rho_{1}$ if and only if any one of the following conditions holds:

(1) for any $\alpha>\rho_{1}$ and all sufficiently large $r, \Phi_{r}(w)<r^{\alpha}$, while for any $\beta<\rho_{1}$ there exist arbitrarily large $r$ such that $\Phi_{r}(w)>r^{\beta}$;

(2) the integral $\int_{1}^{\infty}\left[\Phi_{r}(w) / r^{\kappa+1}\right] d r$ converges for $\kappa>\rho_{1}$ and diverges for $\kappa<\rho_{1}$;

(3) the integral $\int_{1}^{\infty} r^{-*} d \Phi_{r}(w)$ converges $\left({ }^{11}\right)$ for $\kappa>\rho_{1}$ and diverges for $\kappa<\rho_{1}$.

Assertions (1) and (2) have been proved by Nevanlinna [1, pp. 207-208], and for (3) we integrate by parts between the limits 1 and $R(>1)$ and observe that the terms which result are those considered in (1) and (2). Combining Corollary 2.1 with Theorem 5 shows that in all cases $\rho_{1}(w) \leqq \rho(w)$.

3. The Weierstrass representation theorem. Genus. Canonical integrals. Corresponding to the Weierstrass theorem asserting the existence of an entire function with prescribed zeros (not clustering at any finite point), we have Theorem 1 of (I), due to Brelot. However, more explicit information is

(11) The integration here is, of course, to be carried out with respect to $r$. 
at hand in the integral representation of entire $\delta$-subharmonic functions analogous to the Weierstrass infinite product representation for entire functions $\left({ }^{12}\right)$.

Let us denote by $R(z)$ the real part of $z$, and by the integral sign, without indication of the set of integration, the integral over the entire plane $\mathcal{E}$. If $m$ is a mass distribution of finite total variation, vanishing on some neighborhood $N_{\delta}(0)$, then for $p$ any positive integer the function $H$ defined on $\varepsilon$ by

$$
H(z)=\int R\left[(z / \zeta)+(1 / 2)(z / \zeta)^{2}+\cdots+(1 / p)(z / \zeta)^{p}\right] d m(\zeta)
$$

is clearly a harmonic polynomial of degree not exceeding $p$. Applying this fact leads, just as in the classical case, to

THEOREM 6. If $m$ is a mass distribution vanishing on some $N_{\delta}(0)$, then there exists a nondecreasing integral-valued step function $s$ on $(0, \infty)$ such that $\int_{1}^{\infty}(r / t)^{s(t)+1} d \Phi_{t}$ converges for all $r>0$. For $s$ any such function the integral

$$
-\int\left\{\log |1-z / \zeta|+R\left[(z / \zeta)+(1 / 2)(z / \zeta)^{2}+\cdots\right.\right.
$$

$$
\left.\left.+\frac{1}{s(|\zeta|)}(z / \zeta)^{s(|\zeta|)}\right]\right\} d m(\zeta)
$$

with the polynomial part replaced by 0 wherever $s(|\zeta|)=0$, converges uniformly for $z$ on compact sets and defines a complete entire $\delta$-subharmonic function having the mass distribution $m$. Moreover, the corresponding integral with respect to $|d m(\zeta)|$ converges absolutely, so that the integral in (3.1) can be interpreted either as an improper integral or as an actual Radon-Stieltjes-Lebesgue integral over $\mathcal{E}$.

The expression (3.1), yielding an entire $\delta$-subharmonic function in terms of a step function $s$ and a prescribed mass distribution $m$, will be called the Weierstrass integral for $m$ and $s$. Its integrand, the Weierstrass kernel, will be denoted by $E(z / \zeta, s)$.

Corollary 6.1. (Weierstrass REPRESENTATION THEOREM). If $w$ is an entire $\delta$-subharmonic function with mass distribution $m$, then to each $\delta>0$ there correspond a step function $s$ and an entire harmonic function $h$ such that

$$
w(z)=h(z)-\int_{N_{\delta}(0)} \log |z-\zeta| d m(\zeta)-\int_{\varepsilon-N_{\delta}(0)} E(z / \zeta, s) d m(\zeta)
$$

holds on the domain of $w$.

(12) A representation of this sort has been given by Transue [1] for entire subharmonic functions. 
Closely related to the concept of exponent of convergence is that of "genus" of a mass distribution.

Definition 3. By the genus of a mass distribution $m$ on $\varepsilon$ we mean the least non-negative integer $p$ (in the extended sense) for which the integral $\int_{1}^{\infty} r^{-p-1} d \Phi_{r}$ converges.

If the mass distribution $m$ is that of an entire $\delta$-subharmonic function $w$, we shall denote the genus also by $p(w)$.

THEOREM 7. For w any entire $\delta$-subharmonic function,

$$
\rho_{1}(w)-1 \leqq p(w) \leqq \rho_{1}(w) \leqq \rho(w) .
$$

Although the function $s$ of Theorem 6 is not unique, we see that it can be specified as the genus whenever the given mass distribution has finite exponent of convergence.

For $m$ a mass distribution of finite genus $p$ and vanishing on some $N_{\delta}(0)$ we shall refer to $-\int E(z / \zeta, p) d m(\zeta)$ as the canonical integral for $m$. The classical inequality $|E(z / \zeta, p)| \leqq 2|z / \zeta|^{p+1}$ which holds for $|z / \zeta| \leqq 1 / 2$ then leads to the following inequality for canonical integrals: for $z$ on $S_{R / 2}(0)$

$$
\int_{\varepsilon_{-N_{R}(0)}}|E(z / \zeta, p)| \cdot|d m(\zeta)| \leqq 2|z|^{p+1} \int_{R}^{\infty} t^{p-1} d \Phi_{t}
$$

With this at our disposal it is not difficult to prove

TheORem 8. Let $m$ be a mass distribution possessing a finite exponent of convergence and vanishing on some $N_{\delta}(0)$. If $w$ is the function defined by the canonical integral for $m$, then $w$ is a complete entire $\delta$-subharmonic function for which $\rho(w)=\rho_{1}(w)$.

A mass distribution on $\mathcal{E}$ admits a potential if and only if $\int_{1}^{\infty} \log r d \Phi_{r}$ converges. Hence, if a mass distribution admits a potential, its exponent of convergence must vanish, and there follows

Theorem 9. For $w$ a potential, $\rho(w)=\rho_{1}(w)=p(w)=0$.

Applying the Weierstrass representation theorem, we then have

TheOREM 10. If $m$ is a mass distribution having exponent of convergence $\rho_{1}$, there exists an entire $\delta$-subharmonic function having $m$ as its mass distribution and $\rho_{1}$ as its order.

In particular, a necessary and sufficient condition that there exist an entire $\delta$-subharmonic function of finite order having a prescribed mass distribution $m$ is that $m$ have finite exponent of convergence.

4. Functions of finite order. The Hadamard decomposition theorem. Employing the terminology of the theory of entire functions, we refer to the special case of the Weierstrass representation theorem for functions of finite 
order as the "Hadamard decomposition theorem." This theorem and its corollaries play a central role in the theory of functions of potential type.

It is readily shown that the coefficients in the Fourier expansion

$$
h\left(r e^{i \theta}\right)=\sum_{k=0}^{\infty}\left(a_{k} \cos k \theta+b_{k} \sin k \theta\right) r^{k} \quad\left(b_{0} \text { being taken as } 0\right)
$$

of an entire harmonic function $h$ satisfy the inequalities

$$
\left(\left|a_{k}\right|+\left|b_{k}\right|\right) r^{k} \leqq 8 \mu_{r}\left(h^{+}\right)-4 h(0) \quad(k=0,1,2, \cdots) .
$$

In conjunction with Corollary 4.1 this yields

THEOREM 11. If $h$ is an entire harmonic function of finite order, then $h$ is a polynomial of degree $\rho(h)$.

Hence, from the Weierstrass representation theorem we obtain at once

TheOREM 12 (HADAMARD DECOMPOSITION THEOREM). If $w$ is an entire $\delta$-subharmonic function of finite order, having mass distribution of genus $p$, then to each $\delta>0$ there corresponds a harmonic polynomial h of degree not exceeding $\rho(w)$ such that

(4.1) $w(z)=h(z)-\int_{N_{\delta}(0)} \log |z-\zeta| d m(\zeta)-\int_{\varepsilon_{-N_{\delta}(0)}} E(z / \zeta, p) d m(\zeta)$

holds on the domain of $w$.

A simple characterization for the order, provided it is not an integer, follows by invoking Theorem 3 .

COROLlaRy 12.1. If $w$ is an entire $\delta$-subharmonic function of finite nonintegral order, then $\rho(w)=\rho_{1}(w)$.

Observe also that Theorem 12 shows that whenever an entire $\delta$-subharmonic function $w$ of finite order has a mass distribution $m$ admitting a potential, then $w$ is to within an additive harmonic polynomial the potential of $m$.

In general the order does not coincide with the exponent of convergence (e.g. harmonic polynomials). However, we call attention to the following theorem of Brelot [1] and indicate an alternative proof.

THEOREM 13. If $u$ is a non-negative entire subharmonic function of finite order, then $\rho(u)=\rho_{1}(u)$.

Proof. Writing (4.1) for $u$ as $u=h+U$, we have $U \geqq-h$ and therefore $\rho(U) \geqq \rho(h)$. But by Theorem 3, $\rho(u) \leqq \rho(U)=\rho_{1}(u)$, forcing $\rho(u)=\rho_{1}(u)$.

A further application of the Hadamard decomposition theorem results in a representation theorem for entire $\delta$-subharmonic functions of order less 
than one. This has been established for subharmonic functions by Heins [1] directly from the Riesz decomposition theorem $\left({ }^{13}\right)$.

THEOREM 14. If $w$ is an entire $\delta$-subharmonic function of order less than 1 with mass distribution $m$, then to each $\delta>0$ there corresponds a constant $c$ such that

$$
w(z)=c-\int_{N_{\delta}(0)} \log |z-\zeta| d m(\zeta)-\int_{\varepsilon_{-N_{\delta}(0)}} \log |1-z / \zeta| d m(\zeta)
$$

holds on the domain of $w$.

Whenever $w$ is finite at the origin, the potential term can be omitted, the remaining integral being extended over $\varepsilon$. The constant $c$ is then evaluated as $w(0)$.

5. Some remarks on the notion of order in the neighborhood of a point. As noted by Brelot [1], a function $\delta$-subharmonic outside of a disc $S_{r}(0)$ can, by modification of its values in the neighborhood of $C_{r}(0)$, be extended to be $\delta$-subharmonic on the entire plane. Thus, the preceding theory of entire $\delta$-subharmonic functions becomes applicable to functions $\delta$-subharmonic in the neighborhood of infinity, or (by an appropriate inversion) in the neighborhood of any finite point.

A general extension theorem of the sort mentioned above can be readily derived.

THEOREM 15. If $w$ is a function $\delta$-subharmonic on an open set $\Omega$ and $\Omega^{*}$ is an open set with closure in $\Omega$, then there exists an entire $\delta$-subharmonic function coinciding with $w$ on $\Omega^{*}$.

Proof. Let $\Omega^{\prime}$ and $\Omega^{\prime \prime}$ be open sets such that the closure of each of the sets $\Omega^{*}, \Omega^{\prime}, \Omega^{\prime \prime}, \Omega$ lies in the succeeding one. The index $k$ applied to any of these sets will denote its intersection with $N_{k}(0)$.

It is an easy matter to find a function $w_{2}^{*}, \delta$-subharmonic on $N_{2}(0)$ and coinciding with $w$ on $\Omega_{2}^{*}$. For this we take $w_{2}$ as the potential of the mass distribution for $w$ on $\Omega_{4}^{\prime \prime}$ and $h_{2}$ as the harmonic function for which $w=w_{2}+h_{2}$ holds on $\Omega_{4}^{\prime \prime}$. Since $h_{2}$ is continuous on $\bar{\Omega}_{3}^{\prime}$, there is a continuous function $H_{2}$ on $N_{3}(0)$ coinciding with $h_{2}$ on $\Omega_{3}^{\prime}$. The function $h_{2}^{*}$ formed by taking a double areal mean of $H_{2}$ with sufficiently small radius is $\delta$-subharmonic on $N_{2}(0)$ and coincides with $h_{2}$ on $\Omega_{2}^{*}$. Hence, the function $w^{*}=w_{2}+h_{2}{ }^{*}$ has the desired properties.

The theorem will follow by induction if we can show that there exists a function $w_{3}{ }^{*}, \delta$-subharmonic on $N_{3}(0)$, coinciding with $w$ on $\Omega_{3}{ }^{*}$ and with $w_{2}^{*}$ on $N_{1}(0)$. Such a function can, in fact, be constructed in a fashion analogous to that for $w_{2}^{*}$.

(13) See also Brelot [1]. 
Although the extension of a function $w \delta$-subharmonic in the neighborhood of infinity to an entire $\delta$-subharmonic function can be accomplished in a variety of ways, it is evident that any two such extensions differ only by the potential of a mass distribution on a bounded set. Hence, all such extensions have the same order, and it is as this common value that we define the order of $w$. When the point in question is a finite point, rather than the point at infinity, we can without loss of generality take it as the origin. In this case the order is defined as the order of the function obtained from the given function $w$ by a simple inversion.

6. Definition and elementary properties of functions of potential type. Two salient properties of potentials are that they have order zero and that their mass distributions have finite total variation on $\varepsilon$. It would then seem natural to examine the class of functions determined by these two properties.

Definition 4. By a function of potential type we mean a complete entire $\delta$-subharmonic function of order zero whose mass distribution has finite total variation on $\varepsilon$.

The class of all functions of potential type will be denoted by $P$.

In this connection we note that there exist entire $\delta$-subharmonic functions of order zero whose mass distributions do not have finite total variation: for example, the canonical integral of the mass distribution of linear density $1 / x$ on the interval $(1, \infty)$. Furthermore, the class $P$ of all potentials is a proper subclass of $P$, since the latter contains not only all constant functions, but also functions whose mass distributions do not admit potentials. We remark in passing that functions of potential type (other than those which reduce to potentials plus constants) have no proper analogue in complex function theory.

The general theory of entire $\delta$-subharmonic functions leads at once to a representation theorem for functions of potential type.

THEOREM 16. A necessary and sufficient condition that a function w be of potential type is that there exist a constant $c$ and a mass distribution $m$ of finite total variation on $\mathcal{E}$ such that

$$
w(z)=c+\chi(z, m),
$$

where

$$
\chi(z, m)=-\int_{N_{1}(0)} \log |z-\zeta| d m(\zeta)-\int_{\varepsilon_{-N_{1}(0)}} \log |1-z / \zeta| d m(\zeta) .
$$

Moreover, both $c$ and $m$ are uniquely determined by $w$.

This theorem serves essentially to reduce the study of functions of potential type to that of the functions $\chi(z, m)$.

In the course of our investigations use will be made of the following two theorems of Heins [1]. 
THEOREM 17. Let $m$ be a positive mass distribution of genus zero, vanishing on some $N_{\delta}(0)$, and let $u$ be the entire subharmonic function defined by $u(z)$ $=\int \log |1-z / \zeta| d m(\zeta)$. If we set $U(z)=\int_{0}^{\infty} \log |1+z / t| d M_{t}$, then

(1) for all $z$

$$
U(-|z|) \leqq\left\{\begin{array}{l}
U(z) \\
u(z)
\end{array}\right\} \leqq U(|z|), \quad \text { and }
$$

(2) for all $r>0$

$$
\sigma_{r}(u) \leqq U(r)=r \int_{0}^{\infty} \frac{M_{t}}{t(t+r)} d t .
$$

The second theorem of Heins is an extension to subharmonic functions of the classical Wiman theorem.

THEOREM 18. Let $u$ be an entire subharmonic function not identically constant, and let $\iota(r)$ be defined as $\inf _{C_{r}(0)} u$. If $\iota$ is bounded above on $(0, \infty)$, then $\rho(u) \geqq 1 / 2$.

Theorem 17 furnishes an important estimate for the characteristic function of an entire $\delta$-subharmonic function of order less than one.

THEOREM 19. If $w$ is an entire $\delta$-subharmonic function of order less than one and harmonic on some $N_{\delta}(0)$, then there exists a constant $c$ such that

$$
\begin{aligned}
T_{r}(w) & \leqq r \int_{0}^{\infty} \frac{\Phi_{t}(w)}{t(t+r)} d t+c \\
& \leqq \int_{0}^{r} \frac{\Phi_{t}(w)}{t} d t+r \int_{r}^{\infty} \frac{\Phi_{t}(w)}{t^{2}} d t+c .
\end{aligned}
$$

Proof. We are assured by Theorem 35 of (I) of the existence of a constant $C$ such that $T_{r}(w) \leqq T_{r}(u)+T_{r}(v)+C$, where $(u, v)$ is a canonical representation of $w$. An application of Theorem 17 completes the argument.

This result is immediately applicable to functions of potential type $\left({ }^{14}\right)$.

TheOREM 20. If $w$ is a function of potential type harmonic on some $N_{\delta}(0)$, then there exists a constant $c$ such that for $r>0$

$$
T_{r}(w) \leqq \int_{0}^{r}\left[\Phi_{t}(w) / t\right] d t+c .
$$

Proof. It suffices to note that an integration by parts yields

$$
r \int_{r}^{\infty}\left[\Phi_{t}(w) / t^{2}\right] d t=\Phi_{r}(w)+r \int_{r}^{\infty}(1 / t) d \Phi_{t}(w) \leqq \Phi(w) .
$$

(11) Nothing is gained by using instead functions of order less than one having mass distributions of finite total variation, since any such function (if complete) is of potential type. 
Thus, for functions of potential type, Corollary 1.2 can be strengthened as

Corollary 20.1. If $w$ is a function of potential type, then

$$
(1 / 2) \Phi(w) \leqq \lim _{r \rightarrow \infty}\left[T_{r}(w) / \log r\right] \leqq \Phi(w) .
$$

We recover at once the following two theorems of Privaloff [1].

COROLlaRy 20.2. Let w be a complete entire $\delta$-subharmonic function whose mass distribution $m$ admits a potential. A necessary and sufficient condition that $w$ be to within an additive constant the potential of $m$ is that $T_{r}(w)=O(\log r)$ relative to infinity.

COROLLARY 20.3. If $w$ is a complete entire $\delta$-subharmonic function for which $\lim _{r \rightarrow \infty}\left[T_{r}(w) / \log r\right]=0$, then $w$ is identically constant.

A further consequence of Corollary 20.1 is a fundamental criterion for a function to be of potential type.

TheOREM 21. A necessary and sufficient condition that a complete entire $\delta$-subharmonic function $w$ be of potential type is that $\lim _{r \rightarrow \infty}\left[T_{r}(w) / \log r\right]$ exist finitely, or alternatively that $T_{r}(w)=O(\log r)$ relative to infinity.

By virtue of Theorem 4 this leads to a majorization principle in the subharmonic case.

Corollary 21.1. Let $u$ and $v$ be entire subharmonic functions. If $u$ is of potential type and dominates $v$, then $v$ is likewise of potential type.

From the trivial case of $v$ identically constant it is clear that a corresponding theorem is not available for potentials. We can, in fact, make a much stronger statement:

EXAMPLE 1. There exists an entire subharmonic function $v$ dominated by a potential, but such that the mass distribution for $v$ does not admit a potential. To construct such a function, we start with a mass distribution $m$ defined by distributing uniformly on the circles $C_{r_{k}}(0)$ of radius $r_{k}=e^{k}$ the mass $m_{k}=-1 / k^{2}$. Then $m$ has finite total variation but does not admit a potential, and it is obvious that the function $v$ defined by

$$
v(z)=\sum_{k=1}^{\infty}\left|m_{k}\right|(\log |z|-k)^{+}
$$

is a subharmonic function of potential type having $m$ as its mass distribution. Furthermore, $v$ is dominated by the potential $u$ due to the uniform distribution on $C_{1}(0)$ of the total mass $\sum_{k=1}^{\infty} m_{k}$.

It is thus evident that functions of potential type are essential for the solution of the potential theoretic problem of determining the class of entire subharmonic functions dominated by a given subharmonic potential. 
In general, the inequalities of Corollary 20.1 are the best possible, as we find by taking $w$ first as the potential of a unit mass at the origin and then as the potential of the mass distribution consisting of the mass +1 concentrated at the origin and -1 distributed uniformly on $C_{1}(0)$. However, for subharmonic functions the result is considerably sharper.

THEOREM 22. If $u$ is a subharmonic function of potential type, then

$$
\lim _{r \rightarrow \infty}\left[T_{r}(u) / \log r\right]=\Phi(u) .
$$

Proof. Immediate from $\mu_{r}\left(u^{+}\right) \geqq \mu_{r}(u)$, which implies $\lim _{r \rightarrow \infty}\left[T_{r}(u) / \log r\right]$ $\geqq \Phi(u)$.

As a consequence we have a criterion for an entire subharmonic function to be of potential type.

Corollary 22.1. A necessary and sufficient condition for an entire subharmonic function $u$ to be of potential type is that the total mass of $u$ and the total mass of $u^{+}$have a common finite value.

Insight into the situation of Theorem 22 can be gained from the WimanHeins theorem (Theorem 18), which we reword as follows: if $u$ is a nonconstant entire subharmonic function of order less than $1 / 2$ and $\Omega(c)$, for $c$ any constant, is the open set on which $u(z)<c$, then there exists a sequence $\left\{r_{k}\right\}$ of positive numbers diverging to $+\infty$ such that $C_{r_{k}}(0) \cap \Omega(c)=\varnothing$. In particular, the components of $\Omega(c)$ are all bounded regions. Since $u^{+}$is obtained from $u$ by sweeping out the mass of $u$ on the component regions of $\Omega(0)$, it follows that $u$ and $u^{+}$have the same total mass.

Pursuing this topic still further, we use Theorem 4 in conjunction with the identity $\sigma_{r}[\max (u, v)]=\max \left[\sigma_{r}(u), \sigma_{r}(v)\right]$ to infer

THEOREM 23. The upper envelope of two subharmonic functions $u$ and $v$ of potential type is also of potential type, and

$$
\Phi[\max (u, v)]=\max [\Phi(u), \Phi(v)] .
$$

The $\delta$-subharmonic case then follows directly by means of the lattice identity $\max \left(u_{1}-v_{1}, u_{2}-v_{2}\right)=\max \left(u_{1}+v_{2}, u_{2}+v_{1}\right)-\left(v_{1}+v_{2}\right)$.

COROLlaRY 23.1. If $w_{1}$ and $w_{2}$ are functions of potential type, then so is their upper envelope, and

$$
\Phi\left[\max \left(w_{1}, w_{2}\right)\right] \leqq 2\left[\Phi\left(w_{1}\right)+\Phi\left(w_{2}\right)\right] .
$$

7. Vector lattice and Banach space properties. It is clear from Theorem 21 that the class of all functions of potential type is closed under multiplication by reals and under addition, provided the ordinary sum is replaced by its complete extension. Hence, $P$ is a linear space. That $P$ is closed under the operations of taking upper envelopes, lower envelopes, and absolute values follows from Corollary 23.1, and we can summarize our findings as 
THEOREM 24. The family $\mathcal{P}$ of all functions of potential type constitutes a vector lattice over the real field with respect to (i) the operations of multiplication of functions by reals and of addition of functions (followed by taking the complete extension) and (ii) the usual ordering ( $\geqq)$ for functions.

A general norm on $P$ can now be defined in terms of a class of linear functionals.

Definition 5. We denote by $\mathcal{F}$ the class of all linear functionals $F$ on $\mathcal{P}$ satisfying

(1) $F(1)=1$, and

(2) for $w_{k}(z)=\chi\left(z, m_{k}\right),\left\{m_{k}\right\}$ being any sequence of mass distributions of finite total variation on $\varepsilon, \lim _{k \rightarrow \infty} \Phi\left(w_{k}\right)=0$ implies $\lim _{k \rightarrow \infty} F\left(w_{k}\right)=0$.

Since the choice of $F(w)$ as the constant $c$ in the representation (6.1) yields an admissible functional, the class $\mathcal{F}$ is trivially nonvacuous.

It is readily verified that the expression (1.1) for $F \in \mathcal{F}$ defines a valid norm on $P$. The norm depends, of course, on the particular functional $F$ employed, and in the event of possible ambiguity will be referred to as the $F$-norm and denoted by $\|w\|_{F}$. However, the following theorem, a direct consequence of Definition 5, shows that convergence in one norm implies convergence in all norms.

ThEOREM 25. A necessary and sufficient condition for $\left\{w_{k}\right\}$ to converge strongly in $P$ to $w$ is that $\lim _{k \rightarrow \infty} \Phi\left(w_{k}-w\right)=0$ and $\lim _{k \rightarrow \infty} c_{k}=c$, the c's being those of the representation (6.1) for $w_{k}$ and $w$.

In what ensues we shall (as above) suppose $P$ to be normed according to (1.1) for some $F \in \mathcal{F}$.

Before undertaking the proof of our main theorem, asserting that $P=P^{\sharp}$, we state two thoroughly elementary preliminary lemmas. The first deals with a lower semi-continuity property of the functional $\Phi$ and is a special case of formula (19), p. 123, of Banach [1].

LEMмA 1. If $\left\{w_{k}\right\}$ is a sequence of entire $\delta$-subharmonic functions whose mass distributions converge weakly to that of an entire $\delta$-subharmonic function $w$, then

$$
\lim _{k \rightarrow \infty} \inf \Phi\left(w_{k}\right) \geqq \Phi(w) .
$$

The second lemma results from a trivial computation.

LEMMA 2. If $w$ is the potential of a mass distribution $m$, then the corresponding constant $c$ in (6.1) is given by

$$
c=-\int_{\varepsilon-N_{1}(0)} \log |\zeta| d m(\zeta),
$$

and conversely. 
With these lemmas in hand we are in a position to prove that the completion in the metric induced by (1.1) of the space of potentials (in fact, of the space of potentials of mass distributions on bounded sets) is exactly the space of functions of potential type.

Theorem 26. $P$ is a Banach space, and moreover $P=P^{t}$.

Proof. Let $\left\{w_{k}\right\}$ be a Cauchy sequence in the norm, so that $\lim _{j, k \rightarrow \infty} \Phi\left(w_{j}-w_{k}\right)=\lim _{j, k \rightarrow \infty} F\left(w_{j}-w_{k}\right)=0$. Then (Birkhoff [1, p. 253]) there is a mass distribution $m$ such that $\lim _{k \rightarrow \infty} m_{k}=m$ and a constant $C$ such that $\lim _{k \rightarrow \infty} F\left(w_{k}\right)=C$. Since Lemma 1 ensures that $m$ has finite total variation on $\varepsilon$, we can define a function $w$ by (6.1), where $c$ is a constant subject to later determination. Use of a similar representation for $w_{k}$ leads at once to $\lim _{k \rightarrow \infty}\left(c_{k}-c\right)=\lim _{k \rightarrow \infty} F\left(w_{k}-w\right)=C-F(w)$, and there remains but to fix $c$ as $\lim _{k \rightarrow \infty} c_{k}$ to establish $\lim _{k \rightarrow \infty}\left\|w_{k}-w\right\|=0$. Hence $P$ is a Banach space.

As indicated in (1.2), any constant is the strong limit of a sequence of potentials. Moreover, any function of the form $w(z)=\chi(z, m)$ is likewise the strong limit of a sequence $\left\{w_{k}\right\}$ of potentials, since such a sequence can be constructed by taking $w_{k}$ as the sum of the potential of $m$ confined to $S_{k}(0)$ and the potential of the uniform distribution of total mass $\pm 1 / k$ on some appropriate circle $C_{r_{k}}(0), r_{k} \geqq 1$ (note Lemma 2). It follows that $P \subset P^{t}$, proving the second assertion.

Non-separability of $P$ is evinced from the fact that for $w_{1}$ and $w_{2}$ the potentials of distinct point unit masses $\left\|w_{1}-w_{2}\right\| \geqq 2$.

To give an idea of the abundance of functionals $F$, we call attention to the following rather general subclass of $\mathcal{F}$.

ThEOREM 27. Let $f$ be a bounded Borel measurable function on $\varepsilon, E$ a bounded set, and $q$ a distribution of the unit mass on $E$ giving rise to a potential bounded on compact sets. Then

$$
F(w)=\int_{E} w d q+\int f d m,
$$

where $m$ is the mass distribution for $w$, defines a linear functional in $\mathcal{F}$.

Proof. Using the representation (6.1) for $w$ and applying Fubini's theorem, we get

$$
F(w)=c+\int(V-\lambda+f) d m,
$$

where $V$ is the potential of $q$ and $\lambda$ is the potential of the uniform distribution of the unit mass on $C_{1}(0)$. The theorem follows at once from the evident boundedness of the integrand.

The natural ordering of $\mathcal{P}$ according to functional values does not result 
in a Banach lattice (Birkhoff $[1$, p. 246]), since functional domination does not imply a corresponding domination for the total variations of the mass distributions $\left({ }^{15}\right)$. For this reason we introduce an ordering $(>)$ defined by

$$
w>0 \text { provided } w \text { has a positive mass distribution and } F(w) \geqq 0 \text {. }
$$

The functional $F$ appearing here will be taken as that employed in defining the norm.

TheOREM 28. Ordered according to (7.2), $P$ is a Banach lattice and, in fact, an $(L)$ space in the sense of Birkhoff $\left({ }^{16}\right)$.

Proof. The space $P$ is clearly partially ordered by $(>)$, so that to prove it a lattice we need only show that each $w \in P$ has a l.u.b. $(>)$ with 0 . For this we choose the constant $c$ in $W(z)=c+\chi\left(z, m^{+}\right)$to yield $F(W)=\max [F(w), 0]$. Then evidently $\left.W=w^{+(}>\right)$, and $\mathcal{P}$ is a lattice. That $P$ is actually a Banach lattice follows from the evaluation of $|w|(>)$ as the function of potential type whose mass distribution is the total variation function for $w$ and for which $F(|w|(>))=|F(w)|$. Finally, it is obvious that $\mathscr{P}$ is an $(L)$ space, i.e. that $w_{1}>0$ and $w_{2}>0$ imply $\left\|w_{1}+w_{2}\right\|=\left\|w_{1}\right\|+\left\|w_{2}\right\|$.

A theorem of Kakutani [2] then assures us that the conjugate of $P$ is an (M) space.

8. Convergence and closure properties. The sweeping out process. Strong convergence is in general much too restrictive for the problems arising in potential theory. It implies convergence of the mass distributions on all Borel sets, whereas we are usually concerned only with weak convergence of the mass distributions.

Fortunately, however, a suitable modification of the underlying class of functionals permits us to define a mode of convergence in $P$ based on weak convergence of the mass distributions.

Definition 6. We denote by $G$ the class of all linear functionals $G$ on $P$ satisfying

(1) $G(1)=1$, and

(2) for $w_{k}(z)=\chi\left(z, m_{k}\right),\left\{m_{k}\right\}$ being any sequence of mass distributions of finite total variation on $\mathcal{E}$, weak convergence of $\left\{m_{k}\right\}$ to 0 implies $\lim _{k \rightarrow \infty} G\left(w_{k}\right)=0$.

Clearly, $G$ is a subclass of $\mathcal{F}$ and is nonvacuous (we can again take $G(w)$ as the constant $c$ in (6.1)).

Definition 7. A sequence $\left\{w_{k}\right\}$ of functions in $P$ will be said to converge $(A)$ to a function $w \in \mathcal{P}$ provided the mass distributions for $\left\{w_{k}\right\}$ converge weakly to that for $w$ and $\lim _{k \rightarrow \infty} G\left(w_{k}\right)=G(w)$ for some $G \in G$.

(15) A more elementary example of a Banach space which is a lattice, but not a Banach lattice, under the natural ordering is the space of functions $f$ of bounded variation on $[0,1]$ with $f(0)=0$, normed by their total variations.

(16) Birkhoff [1, p. 252]. See also Kakutani [1]. 
That the functional $G$ can be chosen with indifference, as regards convergence $(A)$, is an easy consequence of the representation (6.1).

TheOREM 29. If $\left\{w_{k}\right\}$ converges $(A)$ to $w$, then $\lim _{k \rightarrow \infty} G\left(w_{k}\right)=G(w)$ for every $G \in G$.

It follows by taking $G(w)=c$ according to (6.1) that the limit of a given sequence under convergence $(A)$ is unique.

Proceeding now to the development of properties related to convergence (A), we agree that all norms used are to be defined in terms of a functional $G$ in $G$. With this convention it is clear that strong convergence implies convergence $(A)$, and we show in fact that convergence $(A)$ is weaker than the usual weak convergence.

THEOREM 30. If a sequence $\left\{w_{k}\right\}$ converges weakly in $P$ to $w$, then $\left\{w_{k}\right\}$ converges $(A)$ to w, but not conversely.

Proof. For $E$ any Borel subset of $\varepsilon$ let us denote by $\psi_{E}(w)$ the total mass of $w$ on $E$. Then clearly $\left|\psi_{E}(w)\right| \leqq\|w\|$, so that $\psi_{E}$ is a bounded linear functional on $P$. Hence, weak convergence of $\left\{w_{k}\right\}$ to $w$ yields weak convergence of the corresponding mass distributions. Since every $G \in G$ is a bounded linear functional, convergence (A) of $\left\{w_{k}\right\}$ to $w$ is immediate.

To show the converse false, we construct a sequence $\left\{w_{k}\right\}$ converging (A), but not converging weakly, to a function $w$. This is accomplished by setting $w_{k}(z)=\chi\left(z, m_{k}\right)$, where $m_{k}$ is the uniform distribution of the unit mass on $C_{1-1 / k}(0)$, and defining $w$ similarly as $w(z)=\chi(z, m)$ for $m$ the uniform distribution of the unit mass on $C_{1}(0)$. It is apparent that the sequence so constructed converges (A) to $w$. However, choosing $E=N_{1}(0)$, we have $\psi_{E}\left(w_{k}\right)=1$ and $\psi_{E}(w)=0$, so that $\left\{w_{k}\right\}$ cannot converge weakly to $w$.

The counterexample incorporated in the proof of the foregoing theorem actually implies the stronger conclusion that $G$ is not even dense in the conjugate space of $P$.

Among the elementary properties of convergence (A) we remark first of all a familiar lower semi-continuity of the norm, which follows directly from Lemma 1.

THEOREM 31. If $\left\{w_{k}\right\}$ converges $(A)$ to $w$, then

$$
\liminf _{k \rightarrow \infty}\left\|w_{k}\right\| \geqq\|w\| \text {. }
$$

A further elementary property is that of sequential (A)-compactness of the unit sphere in $P$.

THEOREM 32. Every sequence $\left\{w_{k}\right\}$ of functions in $P$ with $\left\|w_{k}\right\| \leqq 1$ has a subsequence converging $(A)$ to a corresponding function w with $\|w\| \leqq 1$. 
Proof. By the boundedness of $\left\{\Phi\left(w_{k}\right)\right\}$ there is a subsequence $\left\{w_{k}^{*}\right\}$ of $\left\{w_{k}\right\}$ such that the corresponding mass distributions $\left\{m_{k}^{*}\right\}$ converge weakly to a mass distribution $m$ of finite total variation. Also, since $\left\{G\left(w_{k}\right)\right\}$ is bounded, we can assume that $\left\{G\left(w_{k}^{*}\right)\right\}$ converges to some number $C$. Determination of the constant $c$ in $w(z)=c+\chi(z, m)$ to yield $G(w)=C$ then ensures convergence $(\mathrm{A})$ of $\left\{w_{k}^{*}\right\}$ to $w$.

The utility of the notion of convergence (A) depends in large measure on the availability of suitable functionals $G$, and it is therefore desirable to have at our disposal an extensive subclass of $G$. To arrive at such a subclass $\left({ }^{17}\right)$, we have only to observe that the function $V-\lambda$ in equation (7.1) tends to zero at infinity.

THEOREM 33. Let $f$ be a continuous function on $\varepsilon_{\text {vanishing at infinity, } E \text { a }}$ bounded set, and $q$ a distribution of the unit mass on $E$ admitting a continuous potential. Then

$$
G(w)=\int_{E} w d q+\int f d m,
$$

where $m$ is the mass distribution for w, defines a linear functional in $G$.

In particular, the choice of $q$ as proportional to Lebesgue measure $a$ (and $f$ as 0 ) gives rise to

CoROLlary 33.1. If $\left\{w_{k}\right\}$ is a sequence of functions of potential type converging $(A)$ to $w$, then

$$
\lim _{\boldsymbol{k} \rightarrow \infty} \int_{E} w_{k} d a=\int_{E} w d a
$$

for every bounded Lebesgue measurable set $E$.

It is important in the applications of Theorem 33 to be able to take $E$ as any Borel set of positive (interior) capacity, and we show in the following lemma that this is possible.

LeMma 3. Every set of positive interior capacity supports a distribution of the unit mass having a continuous potential.

Proof. By virtue of the fact that every set of positive interior capacity contains a compact subset of positive capacity, it suffices to assume the given set $E$ compact. We then take $m$ as the equilibrium distribution $\left({ }^{18}\right)$ of the unit mass on $E$. As is well known, the equilibrium potential is constant on $E-J$,

(17) As we show in $\$ 9$, the subclass constructed below is actually all of $G$. This fact, however, is not nearly as important as the particular form of the functionals (8.1).

(18) The classical theory of the equilibrium distribution for compact sets, as developed by Frostman $[1$, p. 56], plays a fundamental role here. 
where $J$ is a subset of $E$ for which $m(J)=0$. It then follows from the regularity of Borel measures on $\varepsilon$ that there exists an open set $G$ containing $J$ for which $m(E-G)>0$. The mass distribution $q$ obtained by restricting $m$ to the compact set $E-G$ has a potential continuous on this set( $\left.{ }^{19}\right)$, and a theorem of Evans (Frostman [1, p. 26]) allows us to conclude that the potential of $q$ is continuous throughout $\varepsilon$.

A basic convergence theorem for almost $\delta$-subharmonic functions [(I), Theorem 23] has as a special case the theorem: if $\left\{w_{k}\right\}$ is a bounded sequence of functions $\delta$-subharmonic on a region $\Omega$ and having mass distributions of uniformly bounded total variation, then pointwise convergence of $\left\{w_{k}\right\}$ to a function $w$ implies almost $\delta$-subharmonicity of $w$. One might expect from the generalizations of Brelot and Cartan ([Cartan [1]) of the theorem of Rado $[1$, p. $22(3.37)]$ that more can be said about the set $E$ on which $w$ differs from its regularizing $\delta$-subharmonic function than merely that $E$ has measure zero. We shall show, in fact, that the set $E$ has capacity zero. This draws heavily on a result of Choquet [1] to the effect that all Borel sets are capacitable, the proof of which is based on Choquet's elegant abstract development of the theory of capacity.

Our convergence theorem is essentially a fusion of Theorems 29 and 33 with Theorem 23 of (I).

THEOREM 34. Let $\left\{w_{k}\right\}$ be a sequence of quasi $\delta$-subharmonic functions on a region $\Omega$, having mass distributions of uniformly bounded total variation. If the functions $w_{k}$ all lie between two $\delta$-subharmonic functions $\left({ }^{20}\right)$ and converge pointwise quasi everywhere on $\Omega$ to a function $w$, then there exists $a \delta$-subharmonic function $w^{*}$ (the regularization of $w$ ) differing from $w$ at most on a set of capacity zero. Moreover, the mass distributions for $\left\{w_{k}\right\}$ converge weakly to that for $w^{*}$. If, further, all $w_{k}$ are of potential type, then so is $w^{*}$, and $\left\{w_{k}\right\}$ converges $(A)$ to $w^{*}$.

Proof. By Theorem 23 of (I) we know that $\left\{w_{k}\right\}$ converges almost everywhere to a function $w^{*} \delta$-subharmonic on $\Omega$ and that the corresponding sequence $\left\{m_{k}\right\}$ of mass distributions converges weakly to the mass distribution $m$ for $w^{*}$. Let us now take $\omega$ as any neighborhood with $\bar{\omega} \subset \Omega$ and apply the Riesz decomposition theorem to obtain $w_{k}=W_{k}+h_{k}$ and $w^{*}=W+h$ on $\omega$, where the $W$ 's are the potentials of the mass distributions confined to $\omega$ and the $h$ 's are harmonic. Denoting by $\alpha_{r}$ the areal mean operator, we have $\alpha_{r} w_{k}=\alpha_{r} W_{k}+h_{k}$ and $\alpha_{r} w^{*}=\alpha_{r} W+h$. Then the dominated convergence hypothesis yields $\lim _{k \rightarrow \infty} \alpha_{r} w_{k}=\alpha_{r} w^{*}$, and the weak convergence of the mass distributions entails $\lim _{k \rightarrow \infty} \alpha_{r} W_{k}=\alpha_{r} W$. Hence, $h_{k} \rightarrow h$, so that $W_{k} \rightarrow W$ almost

(19) If the sum of two lower semi-continuous functions is continuous, then each of the functions must be continuous.

${ }^{(20)}$ Actually, all that is required of those functions is that they be summable on all compact sets with respect to all mass distributions admitting continuous potentials. 
everywhere on $\omega$. We see further that if $\sigma$ is any closed disc $C \omega$, then the functions $h_{k}\left(=\alpha_{r} w_{k}-\alpha_{r} W_{k}\right)$ are uniformly bounded on $\sigma$, so that the potentials $W_{k}$ all lie between two functions $\delta$-subharmonic on $\sigma$.

Let us suppose that $w$ and $w^{*}$ differ on a set of positive (exterior) capacity. In view of the fact that $w$ and $w^{*}$ are Baire functions, this set is a Borel set and therefore, by the Choquet theorem, has positive interior capacity. A routine argument shows that $\pm\left(w^{*}-w\right)$ must exceed some $\epsilon>0$ on a compact set $E$ of positive capacity, and Lemma 3 then ensures the existence of a distribution $q$ of the unit mass on $E$ having a continuous potential. As is clearly permissible, we shall assume the $\operatorname{disc} \sigma$ (introduced above) to contain $E$.

It is obvious from the choice of $G$ as $G(\phi)=(1 / a(\sigma)) \int_{\sigma} \phi d a$, where $a$ denotes Lebesgue plane measure, that $\left\{W_{k}\right\}$ converges (A) to $W$. Hence, by Theorems 29 and 33 (with $f \equiv 0$ )

$$
\lim _{\boldsymbol{k} \rightarrow \infty} \int_{E} W_{k} d q=\int_{E} W d q=\int_{E} w^{*} d q-\int_{E} h d q .
$$

On the other hand, application of the Lebesgue convergence theorem results in

$$
\lim _{k \rightarrow \infty} \int_{E} W_{k} d q=\int_{E} w d q-\int_{E} h d q,
$$

and we are thus confronted with the untenable conclusion that $\int_{E}\left(w^{*}-w\right) d q$ $=0$. This proves that $w$ can differ from $w^{*}$ only on a set of capacity zero. Our final assertion, that $w^{*}$ is of potential type whenever the functions $w_{k}$ are, follows without difficulty from Theorem 32 .

In the special case when $\left\{w_{k}\right\}$ is monotone increasing and bounded above, the inclusion hypothesis is, of course, automatically fulfilled. Restricting the functions $w_{k}$ still further to be subharmonic yields the theorem of BrelotCartan (Cartan [1, pp. 99, 105]).

CoRollary 34.1. If $\left\{u_{k}\right\}$ is a monotone increasing sequence of subharmonic functions uniformly bounded above on a region $\Omega$, the limit function is quasi subharmonic on $\Omega$.

Proof. It remains to be verified that the total variations of the mass distributions are, at least locally, uniformly bounded. This is readily shown, however, by presuming that $S_{R}(0) \subset \Omega$, fixing $0<r<R$, and computing the total variation on $S_{r}(0)$ of the mass distribution for $u_{k}$ as $\partial^{+} \mu_{r}\left(u_{k}\right) / \partial \log r$. Since the functions $\mu_{t}\left(u_{k}\right)$ are convex in $\log t$ for $t$ on $[0, R]$ and are uniformly bounded above, it is evident that $\left\{\partial^{+} \mu_{r}\left(u_{k}\right) / \partial \log r\right\}$ is bounded.

The case in which $\left\{w_{k}\right\}$ is a decreasing sequence of subharmonic functions of potential type is also of interest, since it furnishes a generalization of theorems of Evans and M. Riesz. 
CoROLlaRy 34.2. If $\left\{u_{k}\right\}$ is a monotone decreasing sequence of subharmonic functions of potential type for which the limit function $u$ is not identically $-\infty$, then $u$ is a subharmonic function of potential type and $\left\{u_{k}\right\}$ converges $(A)$ to $u$.

Proof. The boundedness of $\left\{\Phi\left(u_{k}\right)\right\}$ is an obvious consequence of Corollary 1.1. (Note that the fact that $u$ is of potential type follows from Corollary 21.1, as well as from Theorem 34.)

We see by taking $u_{k}(z)=\max [1,(1 / k) \log |z|]$ that the analogue of Corollary 34.2 for potentials is not a theorem. However, a classical result due to Evans [1, p. 231] states that if the functions $u_{k}$ are all potentials of negative mass distributions on a compact set $K$, then $u$ is likewise the potential of a negative mass distribution on $K$. In the present context this appears as a direct consequence of Corollary 34.2 and Lemma 2.

M. Riesz [1, p. 32] has generalized the theorem of Evans by allowing the $u_{k}$ to be potentials of arbitrary negative mass distributions, in which case $u$ is the sum of a subharmonic potential and a non-negative constant. Here also, the proof can be based on Corollary 34.2, and for this we note

Lemma 4. If $\left\{u_{k}\right\}$ is a sequence of subharmonic potentials converging $(A)$ to $u$, then $u$ is the sum of a subharmonic potential and a non-negative constant.

Proof. Denoting the corresponding mass distributions by $m_{k}$ and $m$ and applying Lemma 2, we find from the weak convergence of $\left\{m_{k}\right\}$ to $m$ that

$$
-\int_{\mathcal{E}_{-N_{1}(0)}} \log |\zeta| d m(\zeta) \leqq \lim _{k \rightarrow \infty}\left[-\int_{\varepsilon_{-N_{1}(0)}} \log |\zeta| d m_{k}(\zeta)\right]=c,
$$

where $c$ is the constant in the representation (6.1) for $u$. Thus, $m$ admits a potential, so that $u$ is to within an additive constant $C$ the potential of $m$. That $C$ is non-negative follows from its evaluation as $C=c+\int \varepsilon_{-N_{1}(0)} \log |\zeta| d m(\zeta)$.

It should be remarked that the theorems of Evans and M. Riesz can be established also by means of criteria due to Brelot [2, p. 308].

One might hope to generalize the theorem of Evans still further by allowing the $u_{k}$ to be subharmonic functions of potential type whose mass distributions admit potentials, but this evanesces.

EXAmple 2. There exists a decreasing sequence $\left\{u_{k}\right\}$ of subharmonic functions of potential type such that (i) the mass distribution for each $u_{k}$ admits a potential and (ii) the limit function $u$ is not identically $-\infty$ but has a mass distribution not admitting a potential. For this we set $m_{j}=1 / j^{2}$ and define the functions $u_{k}$ by

$$
u_{k}(z)=\sum_{j=1}^{k} m_{j}(\log |z|-j)^{+}+\left(\sum_{j=k+1}^{\infty} m_{j}\right)[\log |z|-(k+1)]^{+} .
$$

A review of the present status of the sweeping out process for potentials reveals that it is customary to sweep out the mass either on bounded sets 
or on sets having bounded complements. The superfluous nature of this restriction is made evident in the following theorem, which serves also to extend the sweeping out process to functions of potential type.

THEOREM 35. Let $u$ be a subharmonic function of potential type and $\Omega$ an open set with non-polar complement. Then $u$ admits a least harmonic majorant $h$ on $\Omega$, and there is a unique subharmonic function $u^{*}$ of potential type such that

(1) $u^{*}$ coincides with $h$ on $\Omega$ and

(2) $u^{*}$ coincides with $u$ outside of $\bar{\Omega}$ and on the set of regular boundary points of $\Omega$.

Further, $\Phi\left(u^{*}\right) \leqq \Phi(u)$, and whenever $u$ is a potential, $u^{*}$ is the sum of a potential and a non-negative constant.

Proof. Since the complement of $\Omega$ is non-polar, it contains a compact set $K$ of positive capacity, and this set supports a distribution $q$ of the unit mass admitting a continuous potential. We denote by $u_{k}$ the subharmonic function of potential type obtained by sweeping out the mass of $u$ on $\Omega$ $\cap N_{k}(0)$ and observe that each $u_{k}$ coincides with $u$ quasi everywhere on $K$. Hence, with norms defined in terms of the functional $G(w)=\int_{K} w d q$, we have $\left\|u_{k}\right\|=\|u\|$ for all $k$. Theorem 32 then ensures the existence of a subsequence $\left\{u_{k}^{*}\right\}$ of $\left\{u_{k}\right\}$ converging (A) to a function $u^{*}$, and there follows

$$
\int_{E} u^{*} d a=\lim _{k \rightarrow \infty} \int_{E} u_{k}^{*} d a \quad(a=\text { Lebesgue plane measure })
$$

for every bounded Lebesgue measurable set $E$.

Now, from the classical theory of balayage $\left({ }^{21}\right)$ we know that $\left\{u_{k}\right\}$ increases monotonically to a function $U$ which (i) coincides with $u$ outside of $\bar{\Omega}$ and on the set of regular boundary points of $\Omega$ and (ii) is either harmonic or identically $+\infty$ on $\Omega$. The monotoneity yields

$$
\int_{E} U d a=\lim _{k \rightarrow \infty} \int_{E} u_{k} d a
$$

and allows us to strengthen (a) as

$$
\int_{E} u^{*} d a=\lim _{k \rightarrow \infty} \int_{E} u_{k} d a
$$

thereby ruling out the second possibility in (ii). $U$ is therefore identical on $\Omega$ to the least harmonic majorant of $u$, and an argument of Brelot $[4 ; 5]$ shows that, except for possible modification on the set $J$ of irregular boundary points of $\Omega, U$ is subharmonic on $\varepsilon$. But, from (b) and (c) we perceive that $U$ and $u^{*}$ are equal almost everywhere, so that by subharmonicity they must

(21) See Brelot [3, p. 433]. 
coincide on $\varepsilon-J$. The remaining assertions are easy consequences of the convergence (A) and Lemmas 1 and 4.

Note that this theorem leads at once to the existence of a generalized Green's function for an arbitrary region having non-polar complement.

One further closure property in $P$ seems worth mentioning. By combining Theorem 4 with the criterion of Theorem 21, we find that if $u$ is a subharmonic function of potential type, then the function $\sigma_{r} u$, where $\sigma_{r} u(z)$ $=\max _{C_{r}(z)} u$, is likewise of potential type. Moreover, since $\sigma_{r} u$ dominates the corresponding functions $\mu_{r} u$ and $\alpha_{r} u$ formed by taking circumferential and areal means, respectively, these also must be of potential type. All of these functions converge downward to $u$ as $r$ decreases to 0 , so that we have convergence $(\mathrm{A})$. There follows

THEOREM 36. If $w$ is a function of potential type and $W$ a $k$-fold iteration of the circumferential (or areal) mean of wover circles of radius $r$, then $W$ is a function of potential type. Moreover, if $\left\{W_{k}\right\}$ is a sequence of such functions corresponding to a sequence $\left\{r_{k}\right\}$ of radii decreasing to 0 , then $\left\{W_{k}\right\}$ converges $(A)$ to w.

9. Functional analysis and potential theory. Although there is an isomorphism between the linear space $P$ of all potentials and the linear space of all mass distributions admitting potentials, so that they are abstractly the same, one cannot thereby dismiss potential theory as simply a phase of measure theory. Potential theory deals with the behavior of potentials as point functions, and this behavior cannot be deduced from the algebraic structure of $P$.

An analogous situation is at hand in the theory of functions of potential type. Thus far, our study of $P$ has been undertaken from the potential theoretic viewpoint, but we proceed to show that, as a Banach space, $P$ can be identified with spaces long familiar in functional analysis.

The Banach space $C_{\infty}$ consisting of all continuous functions $f$ on $\varepsilon$ vanishing at infinity, normed by $\|f\|=\max |f|$, is one of the fundamental spaces of classical functional analysis. Spaces of this sort have been exhaustively studied, particularly in recent years (under the more general hypothesis that $\varepsilon$ be a topological space) by E. Hewitt [1]. Among the familiar properties of $C_{\infty}$ is the fact that its conjugate space $C_{\infty}^{*}$ is comprised of all linear functionals $L$ of the form

$$
L(f)=\int f d m,
$$

where $m$ is a mass distribution of finite total variation on $\mathcal{E}$, uniquely determined by $L$. Since

$$
\|L\|=|m|(\boldsymbol{\varepsilon})
$$

(Hewitt [2, p. 459]), $C_{\infty}^{*}$ can be regarded as the space of all mass distributions 
of finite total variation on $\varepsilon$, normed by their total variations. Weak convergence of mass distributions $\left\{m_{k}\right\}$ to $m$, defined by

$$
\lim _{\boldsymbol{k} \rightarrow \infty} \int f d m_{k}=\int f d m \quad \text { for all } f \in C_{\infty},
$$

is then just weak convergence of linear functionals on $C_{\infty}$ (Banach [1, p. 122]).

Let us now consider the Cartesian product space $\Gamma_{\infty}=R \times C_{\infty}$, where $R$ is the real line. The elements of $\Gamma_{\infty}$ are ordered pairs $\phi=(t, f), t$ being a real number and $f$ a function in $C_{\infty}$, and we take as norm

$$
\|\phi\|=\max (|t|, \max |f|) \text {. }
$$

There results a separable Banach space, whose conjugate space $\Gamma_{\infty}^{*}$ is the set of all linear functionals $\Lambda$ of the form

$$
\Lambda(\phi)=c t+\int f d m,
$$

where $c$ is a real number and $m$ is a mass distribution of finite total variation on $\varepsilon$. Both $c$ and $m$ are uniquely determined by $\Lambda$, so that we have $\Gamma_{\infty}^{*}$ $=R \times C_{\infty}^{*}$. Moreover, it is plain that the norm on $\Gamma_{\infty}^{*}$ is given in terms of a generic element $\mu=(c, m)$ as

$$
\|\mu\|=|c|+|m|(\varepsilon) .
$$

From the representation (6.1) we see at once that the space $P$ normed by $\|w\|=|c|+\Phi(w)$ is isomorphic and isometric with $\Gamma_{\infty}^{*}$. That is to say, $P$ and $\Gamma_{\infty}^{*}$ are structurally indistinguishable. Weak convergence of a sequence $\left\{\Lambda_{k}\right\}$ of linear functionals on $\Gamma_{\infty}$ to a linear functional $\Lambda$ is characterized by

$$
\lim _{k \rightarrow \infty}\left(c_{k} t+\int f d m_{k}\right)=c t+\int f d m \quad \text { for all }(t, f) \in \Gamma_{\infty},
$$

which implies $c_{k} \rightarrow c$ and $m_{k} \rightarrow m$ [weakly]. Thus, under the identification of $\mathcal{P}$ with $\Gamma_{\infty}^{*}$, convergence (A) is equivalent to weak convergence of linear functionals on $\Gamma_{\infty}$.

We conclude our remarks with a demonstration that the subclass of $G$ constructed in Theorem 33 is actually all of $G$. This is based on Theorem 8, p. 131 of Banach [1], which ensures that every weakly continuous linear functional $\psi$ on $\Gamma_{\infty}^{*}$ can be expressed as

$$
\psi(\mu)=c t+\int f d m \quad \text { for some }(t, f) \in \Gamma_{\infty} .
$$

With $P$ identified as $\Gamma_{\infty}^{*}, G$ appears as the set of all weakly continuous linear functionals $\psi$ on $\Gamma_{\infty}^{*}$ satisfying the normalizing condition that $\psi(\mu)=1$ when $\mu=(1,0)$. Hence, $G$ consists of all linear functionals of the form 


$$
\psi(\mu)=c+\int f d m \quad \text { for some } f \in C_{\infty}
$$

The functionals constructed according to Theorem 33 have the representation (7.1):

$$
\psi(\mu)=c+\int(V-\lambda+g) d m \quad \text { for some } g \in C_{\infty} .
$$

Our initial assertion now follows from the fact that $V-\lambda$ is a function in $C_{\infty}$.

\section{BIBLIOGRAPHY}

M. G. ARsove

1. Functions representable as differences of subharmonic functions, Trans. Amer. Math. Soc. vol. 75 (1953) pp. 327-365.

2. Thesis, Brown University, 1950.

S. BANACH

1. Théorie des opérations linéaires, Warsaw, 1932.

G. BIRKHOFF

1. Lattice theory, rev. ed., New York, 1948.

M. BRELOT

1. Etude des fonctions sous-harmoniques au voisinage d'un point singulier, Ann. Inst. Fourier vol. 1 (1950) pp. 121-156.

2. Sur le rôle du point d l'infini dans la thêrie des fonctions harmoniques, Ann. Ecole Norm. vol. 61 (1944) pp. 301-332.

3. Fonctions sous-harmoniques et balayage (Part 2), Académie Royale de Belgique vol. 24 (1938) pp. 421-436.

4. Sur la meilleure majorante harmonique d'une fonction sous-harmonique, C. R. Acad. Sci. Paris vol. 205 (1937) p. 12.

5. Sur les meilleures et plus petites majorantes harmoniques des fonctions sous-harmoniques, H. Cartan C. R. Acad. Sci. Paris vol. 205 (1937) pp. 456-457.

1. Theorie du potentiel newtonien: énergie, capacité, suites de potentiels, Bull. Soc. Math. France vol. 73 (1945) pp. 74-106.

2. Thérie générale du balayage en potentiel newtonien, Annales de l'Université Gre noble vol. 22 (1947) pp. 221-280.

G. Choquet

1. Capacibilité. Theorèmes fondamentaux, C. R. Acad. Sci. Paris vol. 234 (1952) pp. 784-786.

G. C. Evans

1. On potentials of positive mass (Part I), Trans. Amer. Math. Soc. vol. 37 (1935) pp. 226253.

\section{O. Frostman}

1. Potentiel d'équilibre et capacité des ensembles, Lund, 1935.

\section{HeINS}

1. Entire functions with bounded minimum modulus; subharmonic function analogues, Ann.

E. HewiTT of Math. vol. 49 (1948) pp. 200-213.

1. Linear functionals on spaces of continuous functions, Fund. Math. vol. 37 (1950) pp. 161-189.

2. Remarks on the inversion of Fourier-Stieltjes transforms, Ann, of Math. vol. 57 (1953) pp. 458-474. 
M. INOUE

1. On the growth of subharmonic functions and its applications to the study of the minimum modulus of integral functions, Journ. Inst. Polytech. Osaka City Univ. vol. 1 (1950) pp. 71-82.

S. KAKUTANI

1. Concrete representation of abstract $(L)$-spaces and the mean ergodic theorem, Ann. of Math. vol. 42 (1941) pp. 523-537.

2. Concrete representation of abstract (M)-spaces, Ann. of Math. vol. 42 (1941) pp. 994-1024.

R. NevanlinNa

1. Eindeutige analytische Funktionen, Berlin, 1936.

E. E. Privaloff

1. A generalization of Jensen's formula. Part I, Izvestia Akad. Nauk vols. 6-7 (1935) pp. 837-847.

T. RADó

1. Subharmonic functions, Berlin, 1937.

M. RIESZ

1. Intégrales de Riemann-Liouville et potentiels, Acta Szeged. vol. 9 (1938) pp. 1-42.

E. C. TITChMaRSH

1. Theory of functions, 2d ed., Oxford, 1939.

W. R. TRANSUE

1. Representation of a subharmonic function in the neighborhood of a point, Amer. J. Math vol. 65 (1943) pp. 335-340.

G. VALIRON

1. Integral functions, London, 1923.

BROWN UNIVERSITY,

Providence, R. I.

UNIVERSITY OF WASHINGTON

SEaTtle, Wash. 